\title{
Knowledge-Aided Non-Homogeneity Detector for Airborne MIMO Radar STAP
}

\author{
Ting WANG, Yongjun ZHAO, Jiantao WANG \\ Zhengzhou Institute of Information Science and Technology, Science Road 62, Zhengzhou, People’s Republic of China \\ wangtingsp@163.com, zhaoyjzz@163.com,18538324298@126.com
}

Submitted December 20, 2015 / Accepted November 8, 2016

\begin{abstract}
The target detection performance decreases in airborne multiple-input multiple-output (MIMO) radar space-time adaptive processing (STAP) when the training samples contaminated by interference-targets (outliers) signals are used to estimate the covariance matrix. To address this problem, a knowledge-aided (KA) generalized inner product non-homogeneity detector (GIP NHD) is proposed for MIMO-STAP. Firstly, the clutter subspace knowledge is constructed by the system parameters of MIMO radar STAP. Secondly, the clutter basis vectors are utilized to compose the clutter covariance matrix offline. Then, the GIP NHD is integrated to realize the effective training samples selection, which eliminates the effect of the outliers in training samples on target detection. Simulation results demonstrate that in non-homogeneous clutter environment, the proposed KA-GIP NHD can eliminate the outliers more effectively and improve the target detection performance of MIMO radar STAP compared with the conventional GIP NHD, which is more valuable for practical engineering application.
\end{abstract}

\section{Keywords}

Airborne multiple-input multiple-output (MIMO) radar, space-time adaptive processing (STAP), knowledge-aid (KA), general inner product nonhomogeneity detector (GIP NHD), outliers

\section{Introduction}

Multiple-input multiple-output (MIMO) radar has been an attractive research field in recent years [1-12]. Compared with traditional radar, relevant researches have demonstrated that MIMO radars own a lot of potential advantages, such as superior angle resolution, increased Doppler resolution, better clutter mitigation capability, etc. By utilizing airborne MIMO radar for the application of ground moving target indication (GMTI) [6-8], the characteristics of both the aperture size and the pulse integration interval can be improved to achieve lower minimum detectable velocity (MDV). Therefore, the specialty of MIMO radars will lead to better GMTI performance, and bring opportunity to the development of space-time adaptive processing (STAP) technique [8-12].

To realize GMTI in strong clutter environment based on STAP technique [13-15], the clutter covariance matrix has to be estimated from the training samples set. Besides, all the training samples should obey the independent and identical distribution (IID), and the required sample number should be at least as double as the system degrees of freedom (DOFs) according to the well-known ReedMallett-Brennan (RMB) rule [16]. In addition, STAP in airborne MIMO radar becomes even more challenging in complexity and convergence, because of the extra DOFs created by the orthogonal waveforms [8-12]. In practice, it is difficult to acquire enough IID samples to ensure the performance of MIMO radar STAP. Moreover, the training samples non-homogeneity will lead to the estimation error of the clutter covariance matrix and severely degrade the performance of MIMO-STAP [11], [17], [18]. Especially, as a classic factor for forming the non-homogeneous clutter environment, when there exist interference-targets (outliers) signals in the training samples set, the specific phenomenon named target self-nulling will be generated [17-22].

Non-homogeneity detector (NHD) [18-22] is wellknown for its ability to improve the target detection performance of STAP in non-homogeneous environment. The generalized inner products (GIP) method [18-22] is a typical NHD for identifying the outliers with non-homogeneity, under the condition on the accurate estimation of the clutter covariance matrix. However, strong outliers may exist in the training samples set, which will result in severe performance degradation of the GIP NHD. Furthermore, if there are more than one strong outlier in the training samples set, the performance of the GIP NHD to determine and eliminate the weaker outliers will as well be deteriorated significantly [21], [22]. Recently, researchers have proposed the knowledge-aided (KA) STAP methods [22-28] to improve the STAP performance and its robustness in the practical application. Thus the prior knowledge, such as the system parameters, can be taken into consideration in the training sample selection, to guarantee that all the chosen samples satisfy the IID property. Reference [22] derived a robust NHD method to eliminate the outliers from the 
training samples set by utilizing the constructed covariance matrix based on the prolate spheroidal wave functions (PSWF). The method can be seen as a KA technique, which is also applicable to MIMO-STAP case with a slight modification. However, the computation and the application of PSWF are somewhat complicated, since it involves time-band-limited sampling theory and the computation of PSWF eigenvector [8], [22].

In this paper, we propose a KA-GIP NHD for airborne MIMO radar STAP, which is very simple and robust in practice. The clutter subspace knowledge is first calculated by the MIMO-STAP system parameters, and then the clutter basis vectors are utilized to construct the corresponding clutter covariance matrix conveniently. Next, the GIP NHD is integrated with KA-clutter covariance matrix to obtain the GIP statistics. Therefore, by comparing the statistics with the setting threshold, the outliers that contaminate the training samples are eliminated. The effectiveness of the proposed KA-GIP NHD for MIMO-STAP is verified by simulation results.

The remainder of the paper is organized as follows. In Sec. 2, we establish the STAP signal model for side-looking airborne MIMO radar. In Sec. 3, a brief review of the conventional GIP NHD is given and the effect of outliers on the conventional GIP NHD is analyzed to formulate the problem. Then in Sec. 4, we present the construction of the knowledge-aided clutter subspace of MIMO radar, and propose the novel GIP NHD for MIMO-STAP based on the KA-clutter subspace. The simulation results are provided in Sec. 5 to show the performance advantage of the KA-GIP NHD for MIMO-STAP over the conventional GIP NHD. In the end, Section 6 summarizes the conclusion of the paper.

Notations. The operations $(\cdot)^{\mathrm{T}},(\cdot)^{\mathrm{H}},(\cdot)^{-1}, \otimes$ denote the transpose, conjugate transpose, inverse, and Kronecker product, respectively. $E[\cdot]$ and $\operatorname{trace}(\cdot)$ denote the expectation and trace of a matrix. $\mathbf{I}_{M}$ denotes the $M \times M$ identity matrix. $\delta(\cdot)$ stands for the unit impulse function, and $\lceil a\rceil$ stands for the smallest integer larger than $a$.

\section{MIMO Radar STAP Signal Model}

Figure 1 presents a sidelooking monostatic MIMO radar system equipped with collocated transmit and receive linear arrays, in which there are $M$ transmit elements with uniform spacing $d_{\mathrm{T}}$ and $N$ receive elements with uniform spacing $d_{\mathrm{R}}$, and sparse coefficient $\alpha=d_{\mathrm{T}} / d_{\mathrm{R}}$, where $d_{\mathrm{R}}=\lambda / 2$ and $\lambda$ is the wavelength. A coherent processing interval (CPI) consists of $K$ pulses with a constant radar pulse repetition interval (PRI) $T$. The radar platform travels at height $H$ and at velocity $V$. The cone angle between the line-of-sight (LOS) and the velocity vector is $\psi$, while $\theta$ and $\varphi$ are the azimuth angle and the elevation angle, respectively. The number of clutter patches which are uniformly distributed in a range cell is $N_{\mathrm{c}}$. The whole space-

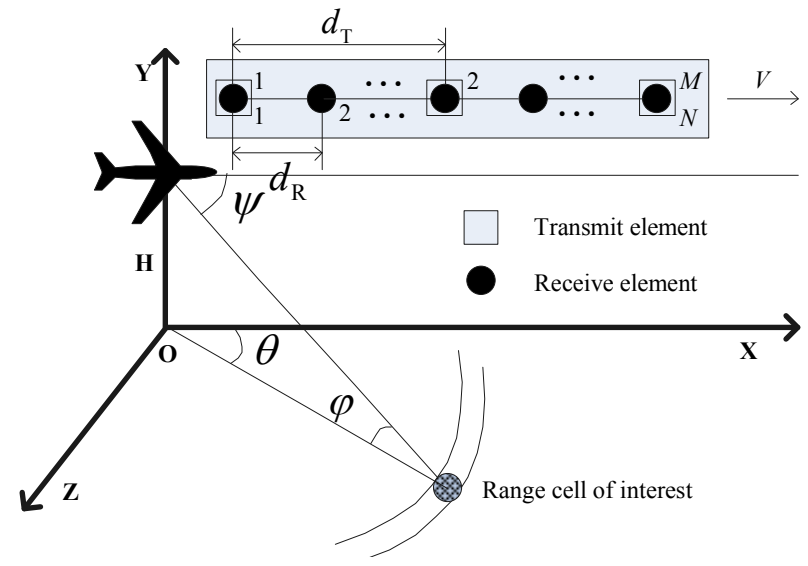

Fig. 1. Configuration of airborne MIMO radar.

time steering vector of MIMO-STAP for a certain clutter patch is written as

$$
\mathbf{v}\left(f_{\mathrm{sc}, i}, f_{\mathrm{dc}, i}\right)=\mathbf{a}_{\mathrm{D}}\left(f_{\mathrm{dc}, i}\right) \otimes \mathbf{a}_{\mathrm{T}}\left(f_{\mathrm{sc}, i}\right) \otimes \mathbf{a}_{\mathrm{R}}\left(f_{\mathrm{sc}, i}\right) .
$$

where

$\mathbf{a}_{\mathrm{D}}\left(f_{\mathrm{dc}, i}\right)=\left[\begin{array}{llll}1 & \mathrm{e}^{\mathrm{j} 2 \pi f_{\mathrm{dc}, i}} & \cdots & \mathrm{e}^{\mathrm{j} 2 \pi(K-1) f_{\mathrm{dc}, i}}\end{array}\right]^{\mathrm{T}}$

$=\left[\begin{array}{llll}1 & \mathrm{e}^{\mathrm{j} 2 \pi \beta f_{\mathrm{sc}, i}} & \cdots & \mathrm{e}^{\mathrm{j} 2 \pi(K-1) \beta f_{\mathrm{sc}, i}}\end{array}\right]^{\mathrm{T}}$,

$\mathbf{a}_{\mathrm{T}}\left(f_{\mathrm{sc}, i}\right)=\left[\begin{array}{llll}1 & \mathrm{e}^{\mathrm{j} 2 \pi \alpha f_{\mathrm{sc}, i}} & \cdots & \mathrm{e}^{\mathrm{j} 2 \pi(M-1) \alpha f_{\mathrm{sc}, i}}\end{array}\right]^{\mathrm{T}}$ and

$\mathbf{a}_{\mathrm{R}}\left(f_{\mathrm{sc}, i}\right)=\left[\begin{array}{llll}1 & \mathrm{e}^{\mathrm{j} 2 \pi f_{\mathrm{sc}, i}} & \cdots & \mathrm{e}^{\mathrm{j} 2 \pi(N-1) f_{\mathrm{sc}, i}}\end{array}\right]^{\mathrm{T}}$ are steering vectors of temporal Doppler, spatial transmit array, and spatial receive array, respectively, $f_{\mathrm{dc}, i}=2 V T \cos \theta_{i} \cos \varphi / \lambda$ and $f_{\mathrm{sc}, i}=d_{\mathrm{R}} \cos \theta_{i} \cos \varphi / \lambda$ are the normalized Doppler and spatial frequencies of the clutter patch at $\theta_{i}$, respectively, $\beta=f_{\mathrm{dc}, i} / f_{\mathrm{sc}, i}=2 V T / d_{\mathrm{R}}$ and $i=1,2, \cdots, N_{\mathrm{c}}$.

The space-time clutter-plus-noise data vector from the $l$ th range cell is denoted as

$$
\begin{aligned}
\mathbf{x}(l) & =\mathbf{x}_{\mathrm{c}}(l)+\mathbf{x}_{\mathrm{n}}(l) \\
& =\sum_{i=1}^{N_{\mathrm{c}}} \rho_{i} \mathbf{v}\left(f_{\mathrm{sc}, i}, f_{\mathrm{dc}, i}\right)+\mathbf{x}_{\mathrm{n}}(l)
\end{aligned}
$$

where $\rho_{i}$ is the reflect coefficient of the $i$ th clutter patch, and $\mathbf{x}_{\mathrm{n}}(l)$ represents the additive Gaussian white noise.

The clutter covariance matrix can be expressed as [13], [27]

$$
\mathbf{R}_{\mathrm{c}}=\sum_{i=1}^{N_{\mathrm{c}}} \sigma_{\mathrm{c}, i}^{2} \mathbf{v}\left(f_{\mathrm{sc}, i}, f_{\mathrm{dc}, i}\right) \mathbf{v}^{\mathrm{H}}\left(f_{\mathrm{sc}, i}, f_{\mathrm{dc}, i}\right)
$$

where $\sigma_{\mathrm{c}, i}^{2}$ is the variance of the $i$ th clutter patch, which is in direct proportion to the radar cross section (RCS) and satisfies $E\left[\left|\rho_{i} \rho_{k}^{*}\right|\right]=\sigma_{\mathrm{c}, i}^{2} \delta(i-k), i, k=1,2, \cdots, N_{\mathrm{c}}$.

Assume the noise obeys the Gaussian distribution and is white in both the spatial and temporal domains, thus the noise covariance matrix can be written as

$$
\mathbf{R}_{\mathrm{n}}=\sigma_{\mathrm{n}}^{2} \mathbf{I}_{K M N}
$$


where $\sigma_{\mathrm{n}}^{2}$ is the variance of the additive white noise.

The fully-adaptive weight vector for MIMO radar STAP can be calculated by

$$
\mathbf{w}=\frac{\mathbf{R}_{\mathrm{c}+\mathrm{n}}^{-1} \mathbf{v}\left(f_{\mathrm{s}, 0}, f_{\mathrm{d}, 0}\right)}{\mathbf{v}^{\mathrm{H}}\left(f_{\mathrm{s}, 0}, f_{\mathrm{d}, 0}\right) \mathbf{R}_{\mathrm{c}+\mathrm{n}}^{-1} \mathbf{v}\left(f_{\mathrm{s}, 0}, f_{\mathrm{d}, 0}\right)}
$$

where $\mathbf{R}_{\mathrm{c}+\mathrm{n}}=\mathbf{R}_{\mathrm{c}}+\mathbf{R}_{\mathrm{n}}$ is the clutter-plus-noise covariance matrix, and $\mathbf{v}\left(f_{\mathrm{s}, 0}, f_{\mathrm{d}, 0}\right)$ is the space-time steering vector of target.

Usually, $\mathbf{R}_{\mathrm{c}+\mathrm{n}}$ is unknown a priori in practice, thus it should be estimated from the adjacent training sample set beside the range cell under test

$$
\hat{\mathbf{R}}=\frac{1}{L} \sum_{l=1}^{L} \mathbf{x}(l) \mathbf{x}^{\mathrm{H}}(l)
$$

where $\mathbf{x}(l)$ denotes the training sample of the $l$ th range cell. Through replacing $\mathbf{R}_{\mathrm{c}+\mathrm{n}}$ by $\hat{\mathbf{R}}$, (5) can be used to calculate the weight vector of the practical MIMO-STAP system. However, with the dimension expansion of the MIMOSTAP system, it is tough to obtain sufficient IID samples in practice, since the practical clutter circumstances are generally non-homogeneous. In particular cases when there exist outliers in the training samples set [11], [17], [18], they will lead to the estimation error of the clutter covariance matrix and seriously degrade the performance of MIMO radar STAP. To solve the non-homogeneity problem, NHDs are utilized to remove outliers from the training samples set before implementing the STAP algorithms to airborne MIMO radar system.

\section{Conventional GIP NHD}

The GIP method [18-22] is a representative criterion of NHDs. We assume the original training samples set $\boldsymbol{\Omega}$ consists of $L$ range cell samples in the adjacent clutter region of the range cell under test, i.e., $\mathbf{\Omega}=\{\mathbf{x}(l)$, $l=1,2, \ldots, L\}$, then we can define the GIP statistics as

$$
\eta_{\mathrm{GIP}}=\mathbf{x}^{\mathrm{H}}(l) \mathbf{R}_{\mathrm{E}}^{-1} \mathbf{x}(l)=\left|\mathbf{R}_{\mathrm{E}}^{-1 / 2} \mathbf{x}(l)\right|^{2}
$$

where $\mathbf{R}_{\mathrm{E}}$ is the test covariance matrix estimated by using the original training samples in set $\boldsymbol{\Omega}$. The GIP statistics can be explained as the inner product of the whitening filter output vector by using $\mathbf{R}_{\mathrm{E}}^{-1 / 2}$, and the expectation of which can be obtained by

$$
E\left[\eta_{\mathrm{GIP}}\right]=\operatorname{trace}\left\{\mathbf{R}_{\mathrm{E}}^{-1} E\left[\mathbf{x}(l) \mathbf{x}^{\mathrm{H}}(l)\right]\right\}=D
$$

where $D=K M N$ denotes the DOFs of MIMO-STAP system. Therefore it can be found from (8) that when the non-homogeneous training sample cannot be effectively whitened by the whitening matrix $\mathbf{R}_{\mathrm{E}}^{-1}$, the GIP statistics in (7) will deviate from expectation $D$. Then the non-homogeneous sample can be easily determined by the deviation level between the GIP statistics and the expectation $D$. Thus, we can eliminate the non-homogeneous training samples when estimating the clutter covariance matrix for MIMO radar STAP.

From the above analysis, we can see that the GIP statistics rely on the accurate estimation of the test covariance matrix $\mathbf{R}_{\mathrm{E}}$. However, different from the homogeneous data shown in (2), the non-homogeneous training sample can be expressed as

$$
\mathbf{x}(l)=\mathbf{x}_{\mathrm{c}}(l)+\mathbf{x}_{\mathrm{n}}(l)+\Delta \mathbf{x}(l)
$$

where $\Delta \mathbf{x}(l)$ is the additional item introduced by the nonhomogeneous environment. Assume the training samples set $\boldsymbol{\Omega}$ contains $P$ non-homogeneous training samples, and then the covariance matrix estimated by using the training samples in $\boldsymbol{\Omega}$ can be written as

$$
\begin{aligned}
\hat{\mathbf{R}}= & \frac{1}{L} \sum_{l=1}^{L} \mathbf{x}(l) \mathbf{x}^{\mathrm{H}}(l) \\
= & \frac{1}{L} \sum_{l=1}^{L-P}\left[\mathbf{x}_{\mathrm{c}}(l)+\mathbf{x}_{\mathrm{n}}(l)\right]\left[\mathbf{x}_{\mathrm{c}}(l)+\mathbf{x}_{\mathrm{n}}(l)\right]^{\mathrm{H}} \\
& +\frac{1}{L} \sum_{l=L-P+1}^{L}\left[\mathbf{x}_{\mathrm{c}}(l)+\mathbf{x}_{\mathrm{n}}(l)+\Delta \mathbf{x}(l)\right]\left[\mathbf{x}_{\mathrm{c}}(l)+\mathbf{x}_{\mathrm{n}}(l)+\Delta \mathbf{x}(l)\right]^{\mathrm{H}} \\
= & \frac{1}{L} \sum_{l=1}^{L}\left[\mathbf{x}_{\mathrm{c}}(l)+\mathbf{x}_{\mathrm{n}}(l)\right]\left[\mathbf{x}_{\mathrm{c}}(l)+\mathbf{x}_{\mathrm{n}}(l)\right]^{\mathrm{H}} \\
& +\frac{1}{L} \sum_{l=L-P+1}^{L}\left[\Delta \mathbf{x}(l) \Delta \mathbf{x}^{\mathrm{H}}(l)+\left(\mathbf{x}_{\mathrm{c}}(l)+\mathbf{x}_{\mathrm{n}}(l)\right) \Delta \mathbf{x}^{\mathrm{H}}(l)+\right. \\
& \left.\Delta \mathbf{x}(l)\left(\mathbf{x}_{\mathrm{c}}(l)+\mathbf{x}_{\mathrm{n}}(l)\right)^{\mathrm{H}}\right] \\
= & \mathbf{R}_{\mathrm{E}}+\Delta \mathbf{R}_{\mathrm{E}} .
\end{aligned}
$$

From (10), we can see that the additional term $\Delta \mathbf{R}_{\mathrm{E}}=$ $\frac{1}{L} \sum_{l=L-P+1}^{L}\left[\Delta \mathbf{x}(l) \Delta \mathbf{x}^{\mathrm{H}}(l)+\left(\mathbf{x}_{\mathrm{c}}(l)+\mathbf{x}_{\mathrm{n}}(l)\right) \Delta \mathbf{x}^{\mathrm{H}}(l)+\Delta \mathbf{x}(l)\left(\mathbf{x}_{\mathrm{c}}(l)+\mathbf{x}_{\mathrm{n}}(l)\right)^{\mathrm{H}}\right]$ will be produced if the training sample set $\boldsymbol{\Omega}$ is contaminated by the outliers, which has a negative effect on the GIP statistics. This will make the conventional GIP NHD difficult to distinguish the non-homogeneous training sample and cause the undetected error. Therefore, the nonhomogeneity of the training samples will also degrade the performance of the conventional GIP NHD in identification of the outliers in the range cell under test [21], [22].

Hence, in order to enhance the robustness of the test covariance and eliminate the negative effects of the outliers on the conventional GIP NHD, we consider utilizing the KA technique in this paper. The MIMO-STAP system parameters, such as platform height and velocity, transmit array element number and spacing, receive array element number and spacing, temporal pulse number and interval, etc., are taken into account as priori knowledge to construct the clutter covariance matrix offline. Then the clutter covariance matrix in the GIP statistics is data-independent and only the homogenous clutter information of the range cell under test is included. Accordingly, the estimation of $\mathbf{R}_{\mathrm{E}}$ is not a requisite, and the knowledge-aided GIP NHD can realize the outliers elimination efficiently. 


\section{Knowledge-Aided GIP NHD for MIMO Radar STAP}

\subsection{KA Clutter Subspace Construction}

Let $\mathbf{C}$ be clutter subspace of MIMO radar STAP. From (3), C can be spanned by all the space-time vectors of the $N_{\mathrm{c}}$ clutter patches in a range cell, that is

$$
\mathbf{C}=\operatorname{span}\left\{\mathbf{v}\left(f_{\mathrm{sc}, i}, f_{\mathrm{dc}, i}\right): f_{\mathrm{dc}, i}=\beta f_{\mathrm{sc}, i}, i=1,2, \cdots, N_{\mathrm{c}}\right\}
$$

The clutter DOFs of MIMO radar, or the rank of the clutter covariance matrix $\mathbf{R}_{\mathrm{c}}$, is denoted as [8]

$$
r_{\mathrm{C}}=\operatorname{rank}\left(\mathbf{R}_{\mathrm{c}}\right)=N+\alpha(M-1)+\beta(K-1) .
$$

Then the space-time vector of the $i$ th clutter patch can be expressed as

$$
\begin{aligned}
& \mathbf{v}\left(f_{\mathrm{sc}, i}, f_{\mathrm{dc}, i}\right)= \\
& =\left[\begin{array}{c}
1 \\
\mathrm{e}^{j 2 \pi f_{\mathrm{sc}, i}} \\
\vdots \\
\mathrm{e}^{j 2 \pi(N-1) f_{\mathrm{sc}, i}} \\
\mathrm{e}^{j 2 \pi \alpha f_{\mathrm{sc}, i}} \\
\vdots \\
\mathrm{e}^{j 2 \pi[\alpha(M-1)+N-1] f_{\mathrm{sc}, i}} \\
\mathrm{e}^{j 2 \pi \beta f_{\mathrm{sc}, i}} \\
\vdots \\
\mathrm{e}^{j 2 \pi[\beta(K-1)+\alpha(M-1)+N-1] f_{\mathrm{sc}, i}}
\end{array}\right]=\mathbf{B}\left[\begin{array}{c}
1 \\
\mathrm{e}^{j 2 \pi f_{\mathrm{sc}, i}} \\
\mathrm{e}^{j 2 \pi 2 f_{\mathrm{sc}, i}} \\
\vdots \\
\mathrm{e}^{j 2 \pi[\beta(K-1)+\alpha(M-1)+N-1] f_{\mathrm{sc}, i}}
\end{array}\right]
\end{aligned}
$$

where $\mathbf{B}$ is a matrix of dimension $K M N \times r_{\mathrm{C}}$, and the $i$ th column and $p$ th row element of $\mathbf{B}$ is denoted as

$$
b_{i, p}=\left\{\begin{array}{cc}
1 & i=(k-1) M N+(m-1) N+n \\
0 & \text { and } p=\beta(k-1)+\alpha(m-1)+n \\
0 & \text { others }
\end{array}\right.
$$

where $k=1,2, \ldots, K, m=1,2, \ldots, M$, and $n=1,2, \ldots, N$. The matrix $\mathbf{B}$ consists of $r_{\mathrm{C}}$ row vectors, that is $\mathbf{B}=$ $\left[\mathbf{b}_{1} \mathbf{b}_{2} \ldots \mathbf{b}_{\mathrm{r}_{\mathrm{C}}}\right]$. From (13), the space-time vector of the $i$ th clutter patch can be expressed as the linear combination of $r_{\mathrm{C}}$ row vectors of $\mathbf{B}$, which means these row vectors can span the clutter subspace of MIMO radar, that is, $\mathbf{C}=$ $\operatorname{span}\left\{\mathbf{b}_{1} \mathbf{b}_{2} \ldots \mathbf{b}_{\mathrm{r}_{\mathrm{C}}}\right\}$. And $\left\{\mathbf{b}_{i}\right\}$ is orthogonal to each other, $i=1,2, \ldots, N_{\text {c }}$. Therefore $\left\{\mathbf{b}_{i}\right\}$ can be seen as the clutter basis vectors for MIMO radar. The orthonormal basis vectors $\left\{\mathbf{b}_{\mathrm{c}, i}\right\}$ for clutter can be derived by the normalizing operation on basis vectors $\left\{\mathbf{b}_{\mathrm{c}, i}\right\}$.

It is should be mentioned that, in the case where $\alpha$ and $\beta$ are non-integers because of the parameter preferences in reality, the clutter DOFs may not be an integer. Then the clutter rank should be set as the smallest integer larger than the non-integer, that is $r_{\mathrm{C}}=\lceil N+\alpha(M-1)+\beta(K-1)\rceil$. In practice, $\mathbf{B}$ can be obtained through offline sampling and stored in memory. Thus, the training data is not required any more to carry out the clutter subspace estimation. In this section, the given clutter subspace is applicable to all the range cells and it can be calculated by the known parameters of transmit array element number $M$, receive array element number $N$, temporal pulse number $K$, coefficients $\alpha$ and $\beta$ according to (14).

\subsection{GIP NHD Based on the KA Clutter Subspace of MIMO Radar}

Based on the theory of the KA clutter subspace presented in the above section, the clutter covariance matrix of MIMO radar can be constructed as

$$
\mathbf{R}_{\mathrm{KA}}=\sum_{i=1}^{r_{\mathrm{C}}} \sigma_{\mathrm{b}, i}^{2} \mathbf{b}_{\mathrm{c}, i} \mathbf{b}_{\mathrm{c}, i}^{\mathrm{H}}
$$

where $\sigma_{\mathrm{b}, i}^{2}$ is the covariance corresponding to each $\mathbf{b}_{\mathrm{c}, i}$ with the average value $\sigma_{\mathrm{b}, i}^{2}=C N R \cdot \sigma_{\mathrm{n}}^{2}=\mathbf{R}_{\mathrm{c}}(1,1) / r_{\mathrm{C}}=\left(\sum_{k=1}^{N_{\mathrm{c}}} \sigma_{\mathrm{c}, k}^{2}\right) / r_{\mathrm{C}}$ to maintain the same clutter-to-noise ratio $(C N R)$ with the clutter covariance matrix $\mathbf{R}_{\mathrm{c}}$ in (3) [13], [14], under the precondition of the same noise variance $\sigma_{\mathrm{n}}^{2}$. Consequently, we can calculate the proposed KA-GIP statistics by substituting (15) into (7)

$$
\eta_{\mathrm{KA}}=\mathbf{x}^{\mathrm{H}}(l) \mathbf{R}_{\mathrm{KA}}^{-1} \mathbf{x}(l) .
$$

It can be observed that the clutter covariance matrix in the proposed KA-GIP statistics merely contains the homogeneous clutter information in the range cell under test. Furthermore, the estimated clutter covariance matrix $\mathbf{R}_{\mathrm{E}}$ will not have any influence on the KA-GIP statistics because the KA-clutter covariance matrix $\mathbf{R}_{\mathrm{KA}}$ has replaced $\mathbf{R}_{\mathrm{E}}$. So the non-homogeneous training samples can be identified and eliminated accurately from estimating the clutter covariance matrix for MIMO-STAP. Thus, the outliers can be eliminated from the training samples set more effectively by the proposed KA-GIP NHD, and the target detection performance of MIMO-STAP will be improved.

It is worth noting that these clutter basis vectors can be calculated offline by the MIMO-STAP system parameters $(M, N, K, \alpha, \beta)$ and memorized in storage. Compared with the PSWF-based clutter subspace knowledge in [22], the construction of the data-independent clutter covariance matrix proposed in this paper is more convenient, though these two methods can achieve approximately the same knowledge-aided effects. Besides, if we constructed the covariance matrix directly by (3) [13], [27], although the discrete representation can approximate the continuous distribution of the real ground clutter, the number and angles of the clutter patches are unknown definitely. This means that the parameters $N_{\mathrm{c}}$ and $\theta_{i}$ are undetermined, and the clutter patches have to be divided and located in the modeling process. Then the construction of the clutter matrix by the columns of $\mathbf{B}$ has lower computational com- 


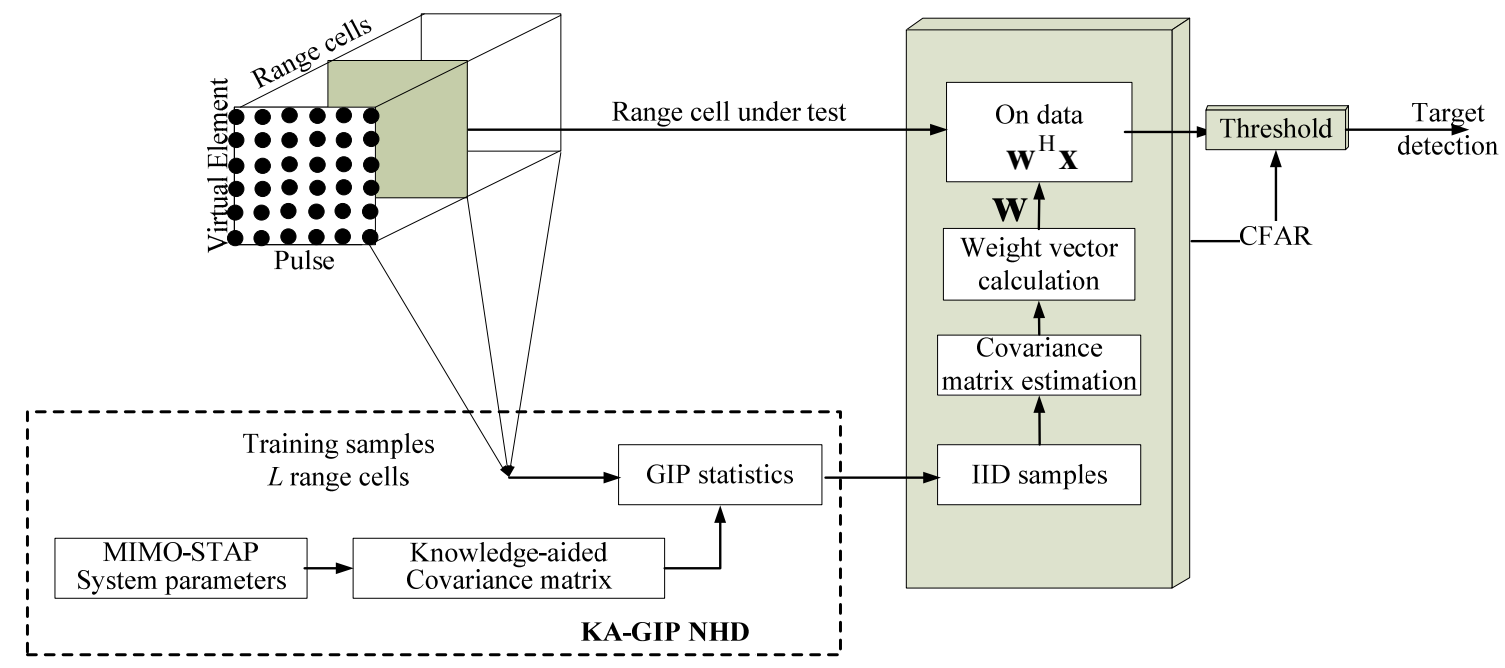

Fig. 2. MIMO-STAP procedure with KA-GIP NHD.

plexity, rather than by (3). Hence for the practical MIMOSTAP system, the proposed KA-GIP NHD is very simple to be executed.

The computational complexity of the proposed KAGIP NHD is herein analyzed. Firstly, in (15), the construction of the KA clutter covariance matrix $\mathbf{R}_{\mathrm{KA}}$ can be realized through $r_{\mathrm{C}}$ multiplications of a $K M N \times 1$ vector $\mathbf{b}_{\mathrm{c}, i}$ by its conjugate transpose $\mathbf{b}_{\mathrm{c}, i}^{\mathrm{H}}$. In this step, the computational cost is $\mathrm{O}\left(r_{\mathrm{C}} K^{2} M^{2} N^{2}\right)$. Then, equation (16) involves the inversion of matrix $\mathbf{R}_{\mathrm{KA}}$, and the computational complexity of $\mathbf{R}_{\mathrm{KA}}^{-1}$ is $\mathrm{O}\left(K^{3} M^{3} N^{3}\right)$. Last, in (16) showing the GIP statistics, the computational burden of $\mathbf{x}^{\mathrm{H}}(l) \mathbf{R}_{\mathrm{KA}}^{-1} \mathbf{x}(l)$ aiming at $L$ samples is $\mathrm{O}\left(L K^{2} M^{2} N^{2}+L K M N\right)$. Taking all these above factors together, we can come to the conclusion that the total computational complexity of the proposed KA-GIP NHD procedure is $\mathrm{O}\left(r_{C} K^{2} M^{2} N^{2}\right)+\mathrm{O}\left(K^{3} M^{3} N^{3}\right)+\mathrm{O}\left(L K^{2} M^{2} N^{2}+L K M N\right)$.

Figure 2 illustrates the corresponding procedure of MIMO radar STAP with this newly-developed NHD. It also should be indicated that the spatial data of MIMOSTAP is equivalent to the $M N \times 1$ virtual array output after matched filtering [8]. The spatial data is corresponding to "Virtual Element" in Fig. 2, i.e., the data of virtual array elements. Meanwhile, the temporal data of MIMO-STAP belongs to each "Pulse" shown in Fig. 2. Afterwards, the spatial data and the temporal data in each range cell are integrated as a whole when processed via the KA-GIP NHD procedure, as we illustrated in the previous principle. In the final target detection step of Fig. 2, CFAR refers to "constant false alarm rate". CFAR detection is a common adaptive algorithm used in radar systems to detect target returns against a background of noise, clutter and interference. According to the changing strength level of the noise, clutter, and interference, the threshold level can be adjusted adaptively to maintain a constant probability of false alarm. Thus the target detection can be efficiently realized by comparing the MIMO-STAP output with the adaptive threshold. And this portion belongs to the next processing step after our proposed NHD.

\section{Simulations}

The basic MIMO-STAP parameters for the simulation are: transmit array element number $M=5$, receive array element number $N=8$, temporal pulse number $K=10$, radar wavelength $\lambda=0.23 \mathrm{~m}$, transmit array element spacing $d_{\mathrm{T}}=0.92 \mathrm{~m}$, receive array element spacing $d_{\mathrm{R}}=0.115 \mathrm{~m}, \alpha=N, \quad \beta=1, \quad$ pulse repetition interval $T=500 \mu \mathrm{s}$, platform velocity $V=115 \mathrm{~m} / \mathrm{s}$, platform height $H=8000 \mathrm{~m}$, clutter patch number $N_{\mathrm{c}}=180$. The CNR is $40 \mathrm{~dB}$. We assume that the cone angle of each outlier is $90^{\circ}$ and the normalized Doppler frequency is 0.25 , which are the same as those of the target. A non-homogeneous clutter scenario is presented, and there are totally $L=500$ range cells in the training set. It should be noted that the value of $L$ is less than double of the system DOFs, i.e. $L<2 M N K$. This implies that the sample number requirement of RMB rule could not be satisfied, and the setting of the training sample number in this simulation accords with the real non-homogeneous clutter scenario. In this simulation four outliers are taken into consideration and Table 1 lists the corresponding outlier parameters.

\begin{tabular}{|c|c|c|}
\hline Outlier number & Range cell & Power relative to noise $[\mathrm{dB}]$ \\
\hline 1 & 100 & 20 \\
\hline 2 & 200 & 30 \\
\hline 3 & 300 & 30 \\
\hline 4 & 400 & 20 \\
\hline
\end{tabular}

Tab. 1. Parameters of outliers.

\section{Experiment 1. Verification of KA clutter subspace basis}

Figure 3 shows the clutter power distribution on each KA clutter subspace basis vector of MIMO radar, which can be expressed as $\mathbf{b}_{\mathrm{c}, i}^{\mathrm{H}} \mathbf{R}_{\mathrm{c}} \mathbf{b}_{\mathrm{c}, i}, i=1,2, \ldots, r_{\mathrm{C}}$. As comparison, Figure 3 illustrates the eigenvalues of $\mathbf{R}_{\mathrm{c}}$. The estimated clutter rank is correctly $N+\alpha(M-1)+\beta(K-1)$. From Fig. 3 we can see that the proposed KA subspace basis vectors possess almost all clutter power, which can be 
utilized to approximate the actual clutter subspace of MIMO radar. Compared with the eigenvalue decomposition (EVD) of the clutter covariance matrix, no samples are needed in obtaining the KA clutter subspace basis vectors, and these basis vectors can be easily updated with the corresponding MIMO radar system parameters.

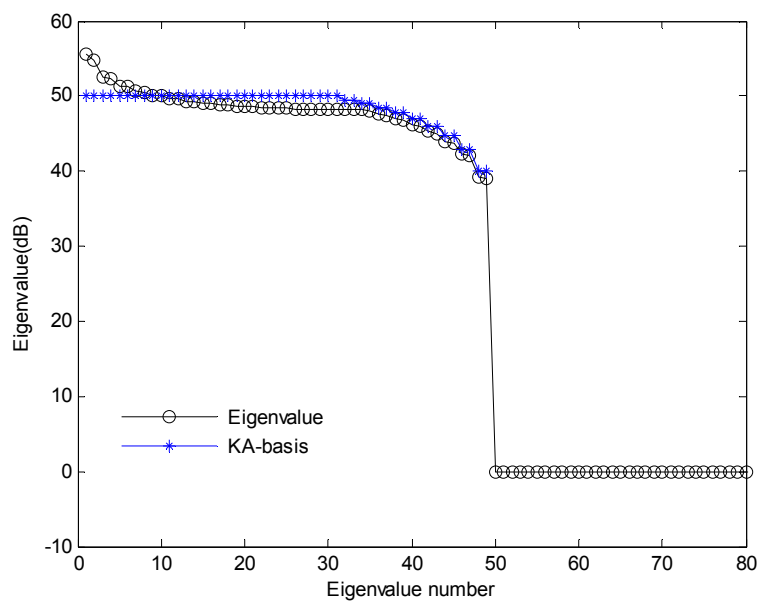

Fig. 3. Clutter power distribution on each KA clutter subspace basis.

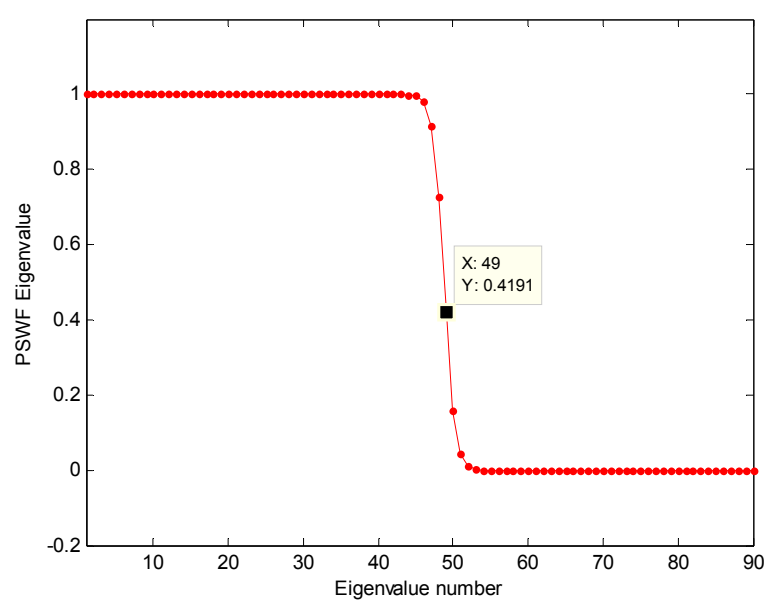

Fig. 4. PSWF eigenvalue.

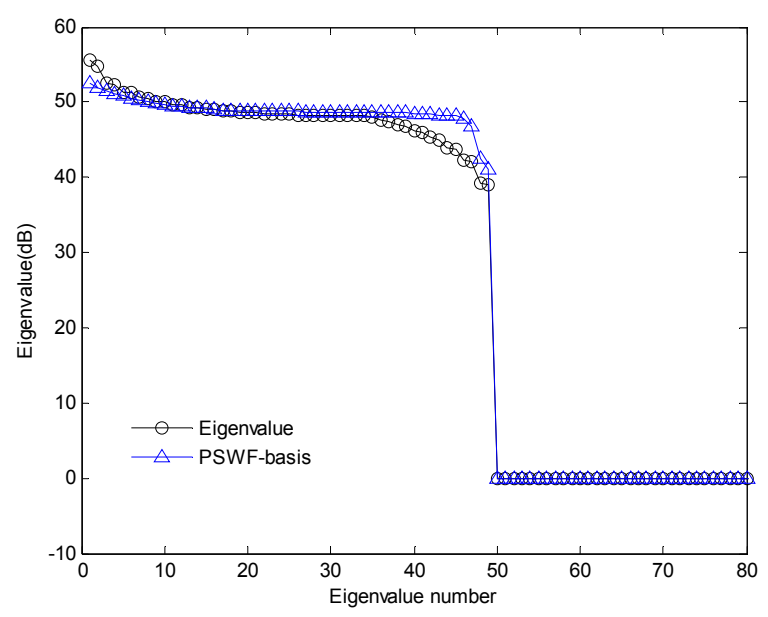

Fig. 5. Clutter power distribution on each PSWF clutter subspace basis.
The eigenvalues of PSWF and the clutter power distribution on each PSWF basis vector are shown in Fig. 4 and Fig. 5, respectively. From the simulation results, it is proved that the PSWF-GIP method in [22] can also be applied to MIMO radar STAP. However, the EVD computation for obtaining PSWF eigenvector has to be implemented when using the PSWF-GIP method with the computational complexity of $\mathrm{O}\left(K^{3} M^{3} N^{3}\right)$. Thus, the KAGIP NHD proposed in this paper is more convenient for application compared with the PSWF-GIP method, although the approximate KA consequence can be attained by both methods.

\section{Experiment 2. Effectiveness and robustness of outliers detection}

The corresponding calculation results of the conventional GIP statistics and the proposed KA-GIP statistics are shown in Fig. 6a) and b), respectively. For each method, the average values are obtained through 100 Monte Carlo trials. It should be noted that the Monte Carlo simulations, as well as other simulation experiments in this paper, are performed by MATLAB software on a computer with CPU Core i7 $2.6 \mathrm{GHz}$ and $8 \mathrm{~GB}$ of RAM. Comparing Fig. 6a) to Fig. 6b), it can be seen that the noise level in the background of Fig. 6b) is obviously much lower, and the proposed KA-GIP NHD improves the average statistics significantly. The conventional GIP NHD only detects two strong outliers, while the KA-GIP NHD has detected all the outliers. It indicates that our proposed NHD is much more effective for MIMO radar STAP than the conventional one.

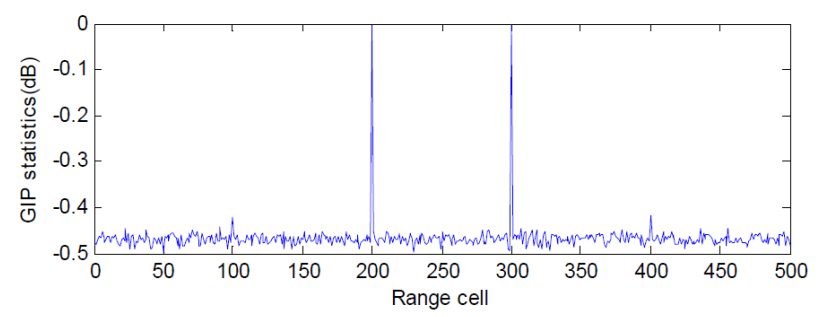

(a)

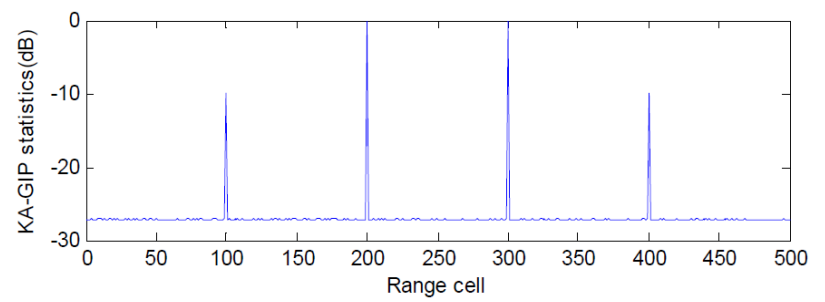

(b)

Fig. 6. Comparison of GIP statistics in non-homogeneous scenario. a) Conventional GIP statistics. b) KA-GIP statistics.

\section{Experiment 3. Clutter suppression and target detection performance of MIMO radar STAP}

The STAP performance is measured by improvement factor $(I F)$, where $I F$ is defined as the ratio of output sig- 
nal-to-clutter-plus-noise-ratio $(S C N R)$ to the input SCNR. Figure 7 shows the $I F$ of the fully-adaptive MIMO-STAP after the outliers in the training samples set are eliminated by the KA-GIP NHD, compared with that for which the conventional GIP NHD is applied to eliminate the outliers. It can be seen that another notch emerges in the outlier Doppler frequency when using the conventional GIP NHD, which is the so-called target self-nulling phenomenon. On the contrary, after using the new NHD proposed in this paper to eliminate the outliers, the MIMO-STAP performance is improved significantly because only IID samples are taken into the STAP weight vector calculation and the effect of outliers has been totally avoided as well as the self-nulling phenomenon.

Suppose there exist two targets which are located in the 130th range cell and the 170th range cell, respectively. The signal-to-noise ratio $(S N R)$ of the target in the 130th range cell is assumed to be $0 \mathrm{~dB}$ while the $S N R$ of the other in the 170th range cell is $-10 \mathrm{~dB}$. The corresponding MIMO-STAP outputs are shown in Fig. 8, after applying the conventional GIP and the KA-GIP NHDs to remove the outliers, respectively. It can be observed from Fig. 8 that by STAP with conventional GIP NHD, the remaining clutter powers in all range cells under test are still too strong, and the weaker target signal in the 170th range cell cannot

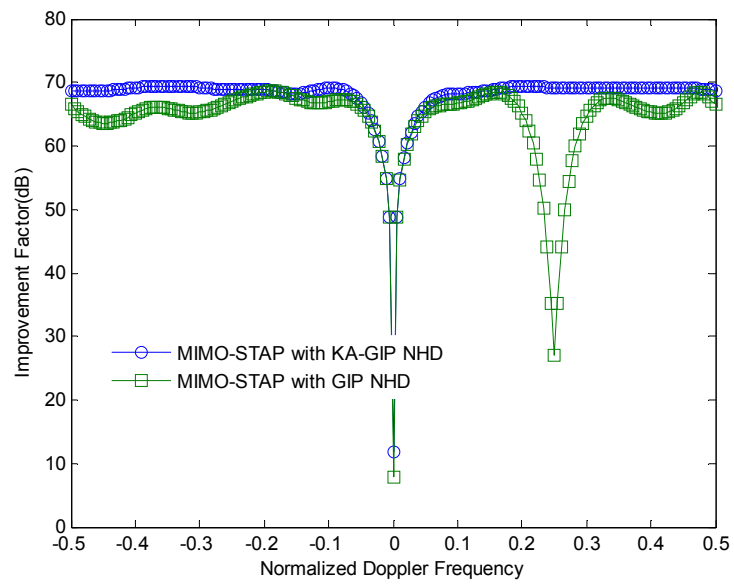

Fig. 7. Comparison of improvement factors.

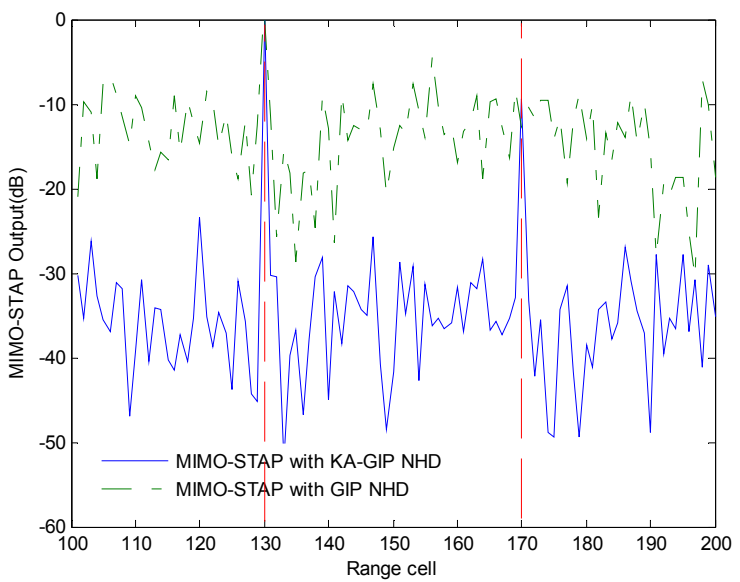

Fig. 8. Comparison of MIMO-STAP filtering outputs. be detected accurately because of the self-nulling effect. At the same time, the peak value of the relatively stronger target in the 130th range cell is also not very distinct. On the other hand, MIMO-STAP with KA-GIP NHD can suppress clutter effectively and detect both targets correctly in the 130th and 170th range cells.

\section{Conclusion}

In this paper, we investigate the non-homogeneity detection technique in airborne MIMO radar STAP, and propose a knowledge-aided GIP NHD for MIMO-STAP. The KA-covariance matrix is constructed to replace the estimated covariance matrix, which is only determined by system parameters and not affected by the outliers at all. Due to this, the outliers can be eliminated more effectively by the novel NHD we put forward, and the target detection performance of MIMO radar STAP in non-homogeneous clutter environments will be improved significantly compared with the conventional GIP NHD. Moreover, the proposed KA-GIP NHD is very convenient to be implemented in practical MIMO-STAP system and has great value for engineering application.

The real scenario can be more complicated and severe than the case we considered in this paper, such as the very rough surface. Under that clutter condition, most regular training samples do not obey the property of IID, and it is difficult to sufficiently estimate the clutter characteristic. Thus, we should deal with the more complicated clutter environment in our future research, and promote relative practical experiment to test our method. Moreover, how to integrate priori knowledge with the real scenario and develop a more effective KA-GIP NHD for MIMO-STAP is also a valuable perspective which will be explored in the future work.

\section{Acknowledgments}

This work was supported by the National Natural Science Foundation of China (61501513).

\section{References}

[1] FISHLER, E., HAIMOVICH, A., BLUM, R., et al. MIMO radar: an idea whose time has come. In Proceedings of the IEEE Radar Conference. Philadelphia (USA), 2004, p. 71-78. DOI: 10.1109/NRC.2004.1316398

[2] HAIMOVICH, A. M., BLUM, R. S., CIMINI, L. J. MIMO radar with widely separated antennas. IEEE Signal Processing Magazine, 2008, vol. 25, no. 1, p. 116-129. DOI: 10.1109/MSP.2008.4408448

[3] LI, J., STOICA, P. MIMO radar with colocated antennas. IEEE Signal Processing Magazine, 2007, vol. 24, no. 5, p. 106-114. DOI: 10.1109/MSP.2007.904812 
[4] FRANKFORD, M. T., STEWART, K. B., MAJUREC, N., et al. Numerical and experimental studies of target detection with MIMO radar. IEEE Transactions on Aerospace and Electronic Systems, 2014, vol. 50, no. 2, p. 1569-1575. DOI: 10.1109/TAES.2014.120180

[5] LI, N., CUI, G., KONG, L., et al. MIMO radar moving target detection against compound-Gaussian clutter. Circuits, Systems, and Signal Processing, 2014, vol. 33, no. 6, p. 1819-1839. DOI: $10.1007 / \mathrm{s} 00034-013-9718-9$

[6] BLISS, D. W., FORSYTHE, K. W. Multiple-input multiple-output (MIMO) radar and imaging: degrees of freedom and resolution. In Proceedings of the 37th Asilomar Conference on Signals, Systems and Computers. Pacific Grove (USA), 2003, p. 54-59. DOI: 10.1109/ACSSC.2003.1291865

[7] BLISS, D. W., FORSYTHE, K. W., DAVIS, S. K., et al. GMTI MIMO radar. In Proceedings of International Waveform Diversity and Design Conference. Kissimmee (USA), 2009, p. 118-122. DOI: 10.1109/WDDC.2009.4800327

[8] CHEN, C. Y., VAIDYANATHAN, P. P. MIMO radar space-time adaptive processing using prolate spheroidal wave functions. IEEE Transactions on Signal Processing, 2008, vol. 56, no. 2, p. 623-635. DOI: 10.1109/TSP.2007.907917

[9] WU, Y., TANG, J., PENG, Y. N. Models and performance evaluation for multiple-input multiple-output space-time adaptive processing radar. IET Radar, Sonar \& Navigation, 2009, vol. 3, no. 6, p. 569-582. DOI: 10.1049/iet-rsn.2008.0025

[10] ZHANG, W., HE, Z.S., LI, J., et al. Beamspace reduced-dimension space-time adaptive processing for multiple-input-multiple-output radar based on maximum cross-correlation energy. IET Radar, Sonar \& Navigation, 2015, vol. 9, no. 7, p. 772-777. DOI: 10.1049/iet-rsn.2014.0226

[11] AHMADI, M., MOHAMED-POUR, K. Space-time adaptive processing for phased-multiple-input-multiple-output radar in the non-homogeneous clutter environment. IET Radar, Sonar \& Navigation, 2014 , vol. 8 , no. 6 , p. $585-596$. DOI: $10.1049 /$ ietrsn.2013.0246

[12] XIANG, C., FENG, D. Z., LV, H. Three-dimensional reduceddimension transformation for MIMO radar space-time adaptive processing. Signal Processing, 2011, vol. 91, no. 8, p. 2121-2126. DOI: 10.1016/j.sigpro.2011.01.017

[13] WARD, J. Space-Time Adaptive Processing for Airborne Radar. Technical Report 1015, Lexington (USA): MIT Lincoln Laboratory, 1994.

[14] GUERCI, J. R. Space-Time Adaptive Processing for Radar. Norwood (USA): Artech House, 2003. ISBN: 1580533779

[15] KLEMM, R. Principles of Space-Time Adaptive Processing. London (UK): IEE, 2002. ISBN: 0863415660

[16] REED, I., MALLETT, J., BRENNAN, L. Rapid convergence rate in adaptive arrays. IEEE Transactions on Aerospace and Electronic Systems, 1974, vol. 10, no. 6, p. 853-863. DOI: 10.1109/TAES.1974.307893

[17] MELVIN, W. L. Space-time adaptive radar performance in heterogeneous clutter. IEEE Transactions on Aerospace and Electronic Systems, 2000, vol. 36, no. 2, p. 621-633. DOI: $10.1109 / 7.845251$

[18] MELVIN, W. L., GUERCI, J. R. Adaptive detection in dense target environments. In Proceedings of IEEE Radar Conference, Atlanta (USA), 2001, p. 187-192. DOI: 10.1109/NRC.2001.922975

[19] SCHOENING， G. N., PICCIOLO， M. L., MILI， L. Improved detection of strong non-homogeneities for STAP via projection statisics. In Proceedings of IEEE International Radar Conference. Arlington (USA), 2005, p. 720-725. DOI: 10.1109/RADAR.2005.1435920
[20] TANG, B., TANG, J., PENG, Y.N. Detection of heterogeneous samples based on loaded generalized inner product method. Digital Signal Processing, 2012, vol. 22, no. 4, p. 605-613. DOI: 10.1016/j.dsp.2012.03.001

[21] RANGASWAMY, M., MICHELS, J. H., HIMED, B. Statistical analysis of the non-homogeneity detector for STAP applications. Digital Signal Processing, 2004, vol. 14, no. 3, p. 253-267. DOI: 10.1016/S1051-2004(03)00021-6

[22] YANG, X. P., LIU, Y.X., LONG, T. Robust non-homogeneity detection algorithm based on prolate spheroidal wave functions for space-time adaptive processing. IET Radar, Sonar \& Navigation, 2013, vol. 7, no. 1, p. 47-54. DOI: 10.1049/iet-rsn.2011.0404

[23] STOICA, P., LI, J., ZHU, X. M., et al. On using a priori knowledge in space-time adaptive processing. IEEE Transactions on Signal Processing, 2008, vol. 56, no. 6, p. 437-444. DOI: 10.1109/TSP.2007.914347

[24] ZHU, X. M., LI, J., STOICA, P. Knowledge-aided space-time adaptive processing. IEEE Transactions on Aerospace and Electronic Systems, 2011, vol. 47, no. 2, p. 1325-1336. DOI: 10.1109/TAES.2011.5751261

[25] TANG, B., TANG, J., PENG, Y.N. Performance of knowledge aided space time adaptive processing. IET Radar, Sonar \& Navigation, 2011, vol. 5, no. 3, p. 331-340. DOI: 10.1049/ietrsn.2010.0131

[26] WU, Y., TANG, J., PENG, Y. N. On the essence of knowledge-aid clutter covariance estimate and its convergence. IEEE Transactions on Aerospace and Electronic Systems, 2011, vol. 47, no. 1, p. 569-585. DOI: 10.1109/TAES.2011.5705692

[27] YANG, Z., DE LAMARE, R. C., LI, X., et al. Knowledge-aided STAP using low rank and geometry properties. International Journal of Antennas and Propagation, 2014, vol. 2014, no. 2, p. 341-346. DOI: $10.1155 / 2014 / 196507$

[28] ZHANG, S., HE, Z., LI, J., et al. A robust colored-loading factor optimization approach for knowledge-aided STAP. In Proceedings of IEEE Radar Conference. Philadelphia (USA), 2016, p. 1-5. DOI: 10.1109/RADAR.2016.7485266

\section{About the Authors ...}

Ting WANG was born in 1988. He is a Ph.D. candidate at Zhengzhou Institute of Information Science and Technology. He received his M.Sc. degree from Hefei Electronic Engineering Institute in 2013. His research interests include space-time adaptive processing and MIMO radar signal processing.

Yongjun ZHAO was born in 1964. He is a professor and Ph.D. advisor at Zhengzhou Institute of Information Science and Technology. He received his M.Sc. degree from Shanghai Jiaotong University in 1992 and his Ph.D. degree from Beijing Institute of Technology in 2008. His research interests include radar signal processing and array signal processing.

Jiantao WANG was born in 1984. He received his M.Sc. degree and Ph.D. degree from the National University of Defense Technology in 2008 and 2014, respectively. He is currently working as a lecturer at Zhengzhou Institute of Information Science and Technology. His research interests include radar signal processing and data processing. 